\title{
LOGARITHMIC SOBOLEV INEQUALITIES FOR THE HEAT-DIFFUSION SEMIGROUP
}

BY

\author{
FRED B. WEISSLER ${ }^{1}$
}

\begin{abstract}
An explicit formula relating the Hermite semigroup $e^{-t H}$ on $R$ with Gauss measure and the heat-diffusion semigroup $e^{t \Delta}$ on $R$ with Lebesgue measure is proved. From this formula it follows that Nelson's hypercontractive estimates for $e^{-t H}$ are equivalent to the best norm estimates for $e^{t \Delta}$ as a map $L^{q}(R)$ into $L^{p}(R), 1<q<p<\infty$. Furthermore, the inequality

$$
\frac{d}{d q} \log \|\phi\|_{q}^{q}<\frac{n}{2 q} \log \left[\frac{q^{2}}{2 \pi n e(q-1)} \cdot \frac{\operatorname{Re}\left\langle-\Delta \phi, J^{q} \phi\right\rangle}{\|\phi\|_{q}^{q}}\right]+\log \|\phi\|_{q},
$$

where $1<q<\infty, J^{q} \phi=(\operatorname{sgn} \phi)|\phi|^{q-1}$, and the norms and sesquilinear form $\langle$,$\rangle are taken with respect to Lebesgue measure on R^{n}$, is shown to be equivalent to the best norm estimates for $e^{t \Delta}$ as a map from $L^{q}\left(R^{n}\right)$ into $L^{p}\left(R^{n}\right)$. This inequality is analogous to Gross' logarithmic Sobolev inequality. Also, the above inequality is compared with a classical Sobolev inequality.
\end{abstract}

1. Introduction. Recent developments in quantum field theory have led to extensive study of the Hermite semigroup. In particular, Nelson's hypercontractive estimates have played a central role. Gross [3] has shown that these estimates are equivalent to a "logarithmic Sobolev inequality". Motivated by the application of probabilistic methods in Nelson's and Gross' work, Beckner [1] has derived sharp forms of the Hausdorff-Young inequality for the Fourier transform and Young's inequality for convolution. These inequalities give the best possible norm estimates for the heat-diffusion semigroup as a map from $L^{q}$ to $L^{p}$.

In this paper we will show that Nelson's hypercontractive estimates for the Hermite semigroup are equivalent to the corresponding norm estimates for the heat-diffusion semigroup. This fact is implicit in Beckner's work: he uses the sharp convolution inequality to prove Nelson's hypercontractive estimates. See Beckner [1, Theorem 5, p. 176]. Also, Brascamp and Lieb [2, $\$ 2.5$ and

Presented to the Society, January 27, 1977; received by the editors November 12, 1976.

AMS (MOS) subject classifications (1970). Primary 47D05; Secondary 46E30, 46E35.

Key words and phrases. Logarithmic Sobolev inequalities, heat-diffusion semigroup, Hermite semigroup, hypercontractivity.

${ }^{1}$ Research supported by a Danforth Graduate Fellowship and a Weizmann Postdoctoral Fellowship. 
Theorem 14] derive the sharp convolution inequality and Nelson's estimates from the same general result. Our proof of the equivalence of the norm estimates for the two semigroups involves an explicit formula relating the two semigroups.

The equivalence of these norm estimates suggests that there is another "logarithmic Sobolev inequality", which is equivalent to the norm estimates for the heat-diffusion semigroup, just as Gross' inequality is equivalent to Nelson's hypercontractive estimates for the Hermite semigroup. This is indeed the case, and in this paper we will derive this inequality and compare it with the classical Sobolev-Nirenberg inequalities.

2. Statement of results. The heat-diffusion semigroup on $R^{n}$ is given by

$$
\begin{aligned}
& \left(e^{t \Delta} \phi\right)(x)=\int_{R^{n}} h_{t}(x-y) \phi(y) d y, \\
& h_{t}(x)=(4 \pi t)^{-n / 2} \exp \left(-|x|^{2} / 4 t\right) .
\end{aligned}
$$

$e^{i \Delta}$ is a contraction $C_{0}$ semigroup on $L^{q}\left(R^{n}, d x\right), 1 \leqslant q<\infty(d x$ denotes Lebesgue measure). We use $D_{q}(\Delta)$ to denote the domain of its generator in $L^{q}\left(R^{n}, d x\right)$. Beckner's convolution inequality [1, Theorem 3, p. 169] implies that for all $\phi \in L^{q}\left(R^{n}\right)$,

$$
\left\|e^{t \Delta} \phi\right\|_{p} \leqslant\left[C_{q} C_{p^{\prime}}\right]^{n}(4 \pi t r)^{-n / 2 r}\|\phi\|_{q^{\prime}},
$$

where $1<q<p<\infty, t>0, p^{-1}+\left(p^{\prime}\right)^{-1}=1, r^{-1}=q^{-1}-p^{-1}$, and $C_{p}$ $=\left[p^{1 / p}\left(p^{\prime}\right)^{-1 / p^{\prime}}\right]^{1 / 2}$. The constant in (1) cannot be improved, as can easily be seen by letting $\phi$ be an arbitrary Gaussian function.

The Hermite semigroup on $R$ is given by

$$
\begin{gathered}
\left(e^{-t H} \phi\right)(x)=\int_{R} k_{\omega}(x, y) \phi(y) d y, \\
k_{\omega}(x, y)=\left[\pi\left(1-\omega^{2}\right)\right]^{-1 / 2} \exp \left(-(\omega x-y)^{2} /\left(1-\omega^{2}\right)\right),
\end{gathered}
$$

where $\omega=e^{-t}$. Let $\mu$ denote the Gauss probability measure on $R$ given by $d \mu(x)=\pi^{-1 / 2} \exp \left(-x^{2}\right) d x$. Then $e^{-t H}$ is a contraction $C_{0}$ semigroup on $L^{q}(\mu), 1 \leqslant q<\infty$; and as before $D_{q}(H)$ is the domain of its generator in $L^{q}(\mu)$. Nelson's hypercontractive estimates [5, Theorem 3] state that for all $\phi \in L^{q}(\mu)$,

$$
\left\|e^{-t H} \phi\right\|_{p(t, q)} \leqslant\|\phi\|_{q}
$$

where $1<q<\infty, t>0$, and $p(t, q)=q+(q-1)\left(e^{2 t}-1\right)$. Here, the norms are taken with respect to the measure $\mu$. The constant 1 in (2) cannot be improved.

In order to state the first theorem, we need to introduce two additional transformations. For a function $\phi$ defined on $R$ and an $\alpha>0$, let $\left(T_{\alpha} \phi\right)(x)=$ 
$\phi(\alpha x)$. Then $T_{\alpha}$ is a map from $L^{q}(R, d x)$ onto itself with $\alpha^{1 / q}\left\|T_{\alpha} \phi\right\|_{q}=\|\phi\|_{q}$. Moreover, one has the formula

$$
e^{t \Delta} T_{\alpha}=T_{\alpha} \exp \left(t \alpha^{2} \Delta\right),
$$

which can be verified by a straightforward calculation. For a function $\phi$ defined on $R$ and a finite $q \geqslant 1$, let

$$
\left(M_{q} \phi\right)(x)=\pi^{-1 / 2 q} \exp \left(-x^{2} / q\right) \phi(x) .
$$

Then $M_{q}$ is an isometric isomorphism of $L^{q}(d \mu)$ onto $L^{q}(d x)$.

THEOREM 1. Let $1<q<p<\infty$ and $t, s>0$. Suppose $p=p(t, q)=q+$ $(q-1)\left(e^{2 t}-1\right)$. Then the heat-diffusion semigroup on $R$ and the Hermite semigroup satisfy the relation

$$
e^{-t H}=\pi^{1 / 2 r} e^{t}(q / p)^{1 / 2} M_{p}^{-1} T_{\beta} e^{s \Delta} T_{\alpha} M_{q},
$$

where $r^{-1}=q^{-1}-p^{-1}$ and

$$
\alpha=\left[\frac{\left(1-\omega^{2}\right) q}{4 s \omega^{2} p}\right]^{1 / 2}, \quad \beta=\left[\frac{4 s q}{\left(1-\omega^{2}\right) p}\right]^{1 / 2}, \omega=e^{-t} .
$$

In particular, it follows that Nelson's hypercontractive estimates (2) are equivalent to the norm estimates (1) for the heat-diffusion semigroup on $R$.

For a positive measure $\nu$, the duality map $J^{q}: L^{q} \rightarrow L^{q^{\prime}}$, where $1<q<\infty$, $q^{\prime}$ conjugate to $q$, is given by $J^{q} \phi=(\operatorname{sgn} \phi)|\phi|^{q-1}$. Also $\langle\phi, \psi\rangle$ denotes the sesquilinear form $\int \phi \bar{\psi} d \nu$, with $\operatorname{Re}\langle$,$\rangle indicating its real part. Finally,$ $\mathscr{D}=\mathscr{D}\left(R^{n}\right)$ is the set of $C^{\infty}$ functions on $R^{n}$ with compact support.

THEOREM 2. The norm estimates (1) for the heat-diffusion semigroup on $R^{n}$ are equivalent to the inequality

(4) $\frac{d}{d q} \log \|\phi\|_{q}^{q} \leqslant \frac{n}{2 q} \log \left[\frac{q^{2}}{2 \pi n e(q-1)} \cdot \frac{\operatorname{Re}\left\langle-\Delta \phi, J^{q} \phi\right\rangle}{\|\phi\|_{q}^{q}}\right]+\log \|\phi\|_{q}$,

which holds for all $\phi$ in $D_{q}(\Delta)$ not identically zero, $1<q<\infty$. The norms and sesquilinear form $\langle$,$\rangle are taken with respect to Lebesgue measure on R^{n}$. Moreover, in order to prove the norm estimates for the heat-diffusion semigroup or the above inequality, it suffices either to verify the norm inequality (1) in the restricted case where $q=2$ and $\phi$ is any nonnegative function in $D$, or to verify the inequality (4) at $q=2$ for all nonnegative functions $\phi$ (not identically 0 ) in o).

REMARKs. (a) If $\phi$ is a Gaussian function, then the two sides of (4) are equal. Thus (4) is a "sharp" inequality.

(b) As we have indicated, the norm estimates (1) have been proved for all $q$ and $p, 1<q<p<\infty$. The point of the latter part of the theorem is that 
they in fact follow from a special case of those estimates, or from a special case of (4).

(c) That (4) is a Lebesgue measure analogue of Gross' logarithmic Sobolev inequality will be clear from the discussion of Gross' inequality in $\$ 5$ below and the proof of Theorem 2 in $\$ 6$ below.

3. Proof of Theorem 1. The proof of Theorem 1 is, of course, "simply a calculation". Since this calculation is somewhat nonroutine, we will show it in reasonable detail.

Let $\omega=e^{-t}$. Then $p=\left(q-1+\omega^{2}\right) / \omega^{2}$. Also let

$$
\sigma=q / \omega p=q \omega /\left(q-1+\omega^{2}\right), \quad \tau=\left(1-\omega^{2}\right) / 4 \omega .
$$

It is then straightforward to check that

$$
-(\omega x-y)^{2} /\left(1-\omega^{2}\right)+y^{2} / q=x^{2} / p-(\sigma x-y)^{2} / 4 \sigma \tau,
$$

from which it follows that

$$
k_{\omega}(x, y) \exp \left(y^{2} / q\right)=\omega^{-1}(q / p)^{1 / 2} \exp \left(x^{2} / p\right) h_{\sigma \tau}(\sigma x-y),
$$

where $k_{\omega}$ and $h_{t}$ are the kernels for the Hermite and heat-diffusion semigroups, respectively. Thus,

$$
\begin{aligned}
\left(e^{-t H} \phi\right)(x) & =\int k_{\omega}(x, y) \phi(y) d y \\
& =\pi^{1 / 2 q} \int k_{\omega}(x, y) \exp \left(\frac{y^{2}}{q}\right)\left(M_{q} \phi\right)(y) d y \\
& =\pi^{1 / 2 q} \omega^{-1}\left(\frac{q}{p}\right)^{1 / 2} \exp \left(\frac{x^{2}}{p}\right) \int h_{\sigma \tau}(\sigma x-y)\left(M_{q} \phi\right)(y) d y \\
& =\pi^{1 / 2 q} \omega^{-1}\left(\frac{q}{p}\right)^{1 / 2} \exp \left(\frac{x^{2}}{p}\right)\left(e^{\sigma \tau \Delta} M_{q} \phi\right)(\sigma x) ;
\end{aligned}
$$

and so

$$
e^{-t H}=\pi^{1 / 2 q-1 / 2 p} \omega^{-1}(q / p)^{1 / 2} M_{p}^{-1} T_{\sigma} e^{\sigma \tau \Delta} M_{q} .
$$

Now $e^{\sigma \tau \Delta}=T_{1 / \alpha} e^{s \Delta} T_{\alpha}$ where $s \alpha^{2}=\sigma \tau$. Substituting this into (5), we get (3).

(3) leads to an explicit relationship between the operator norm of $e^{s \Delta}$ : $L^{q}(d x) \rightarrow L^{p}(d x)$ and the operator norm of $e^{-t H}: L^{q}(d \mu) \rightarrow L^{p}(d \mu)$, where $p=p(t, q)$. Let $B_{1}$ and $B_{2}$ be these two norms, respectively. Since $M_{q}$ : $L^{q}(d \mu) \rightarrow L^{q}(d x)$ and $M_{p}: L^{p}(d \mu) \rightarrow L^{p}(d x)$ are invertible isometries, and since $T_{\alpha}$ and $T_{\beta}$ scale the norms on $L^{q}(d x)$ and $L^{p}(d x)$ by appropriate factors, it follows that 


$$
\begin{aligned}
B_{1} / B_{2} & =\left(\omega / \pi^{1 / 2 r}\right)(p / q)^{1 / 2} \beta^{1 / p} \alpha^{1 / q} \\
& =(4 \pi s)^{-(1 / q-1 / p) / 2}(p / q)^{(1-1 / q-1 / p) / 2} \omega^{1-1 / q}\left(1-\omega^{2}\right)^{(1 / q-1 / p) / 2} .
\end{aligned}
$$

If we can show that this last expression equals $C_{q} C_{p^{\prime}}(4 \pi s r)^{-1 / 2 r}$ (recall $r^{-1}=q^{-1}-p^{-1}$ and $p^{\prime}$ is conjugate to $p$ ), it will follow that (1), which gives the value of $B_{1}$, and (2), which gives the value of $B_{2}$, imply each other. (Observe that for arbitrary $q$ and $p$ with $1<q<p<\infty$, there is a positive $t$ such that $p=p(t, q)$.)

Now, using $p^{\prime}=p /(p-1)$ and $q^{\prime}=q /(q-1)$, we have that

$$
\begin{aligned}
C_{q} C_{p^{\prime}}\left(\frac{1}{q}-\frac{1}{p}\right)^{(1 / q-1 / p) / 2} & \\
& =\frac{q^{1 / 2 q}}{\left(q^{\prime}\right)^{1 / 2 q^{\prime}}} \cdot \frac{\left(p^{\prime}\right)^{1 / 2 p^{\prime}}}{p^{1 / 2 p}} \cdot\left(\frac{p-q}{p q}\right)^{(1 / q-1 / p) / 2} \\
& =\left(\frac{p}{q}\right)^{(1-1 / p-1 / q) / 2} \cdot \frac{(q-1)^{1 / 2 q^{\prime}}}{(p-1)^{1 / 2 p^{\prime}}} \cdot(p-q)^{1 / 2 q-1 / 2 p}
\end{aligned}
$$

Furthermore, since $p=p(t, q)$, it follows that $p-q=(q-1)\left(1-\omega^{2}\right) / \omega^{2}$ and $p-1=(q-1) / \omega^{2}$. Substituting these expressions into the final part of (6), we obtain the desired result.

This concludes the proof of Theorem 1.

4. A differentiation formula. Before proving Theorem 2, we must re-prove a key differentiability lemma of Gross [3, Lemma 1.1, p. 1065]. Gross proves the lemma in the context of a probability measure, and we need the result for a general positive measure. Accordingly, in this section we prove the following proposition.

Proposition 1. Let $\nu$ be a positive measure on a set $X$. Suppose $1<q<p$ $<\infty$ and $a<b$. For each $t \in(a, b)$ let $\phi(t)$ be a complex function on $X$ (not identically zero) such that the curve $t \mapsto \phi(t)$ is continuously differentiable on $(a, b)$ into $L^{s}(\nu)$ for each $s \in(q, p)$, with derivative $\phi^{\prime}(t)$. Then the function $F(t, s)=\|\phi(t)\|_{s}$ is continuously differentiable on $(a, b) \times(q, p)$ with partial derivatives:

$$
\begin{gathered}
\frac{\partial F}{\partial t}(t, s)=\|\phi(t)\|_{s}^{1-s} \operatorname{Re}\left\langle\phi^{\prime}(t), J^{s} \phi(t)\right\rangle, \\
\frac{\partial F}{\partial s}(t, s)=s^{-1}\|\phi(t)\|_{s}^{1-s} \int|\phi(t)|^{s} \log |\phi(t)| d \nu \\
-s^{-1}\|\phi(t)\|_{s} \log \|\phi(t)\|_{s},
\end{gathered}
$$

where $J^{s}$ is the duality map defined in $\S 2$ and $0^{s} \log 0$ is taken to be 0 . 
Proof. We first show that the partial derivatives exist and are given by (7) and (8). It is well known (Mazur [4, p. 132]) that the $L^{s}$ norm is continuously Frechet differentiable on $L^{s}-\{0\}$ with derivative at $\phi$ given by

$$
\left(D\|\cdot\|_{s}\right)_{\phi}(\psi)=\|\phi\|_{s}^{1-s} \operatorname{Re}\left\langle\psi, J^{s} \phi\right\rangle .
$$

(7) now follows from the chain rule.

If $\phi$ is in $L^{s}$ for all $s \in(q, p)$, then $\|\phi\|_{s}^{s}$ is a differentiable function of $s$ and

$$
\frac{d}{d s}\|\phi\|_{s}^{s}=\int|\phi|^{s} \log |\phi| d \nu .
$$

This follows by differentiating under the integral, which can be justified here with the dominated convergence theorem since

$$
|\log | \phi|| \leqslant(e \alpha)^{-1}\left[|\phi|^{\alpha}+|\phi|^{-\alpha}\right]
$$

for all $\alpha>0$. (8) now follows by elementary calculus.

To show that $F(t, s)$ is continuously differentiable, it remains to show that its partial derivatives are jointly continuous in $t$ and $s$. It is straightforward to see that $F(t, s)=\|\phi(t)\|_{s}$ itself is jointly continuous. Thus it suffices to show that $\left\langle\phi^{\prime}(t), J^{s} \phi(t)\right\rangle$ and $\int|\phi(t)|^{s} \log |\phi(t)| d \nu$ are jointly continuous in $t$ and $s$. We accomplish this in the following lemma, which proves a bit more.

LEMMA 1. Let $\phi(t)$ and $\psi(t)$ be continuous curves on $(a, b)$ into $L^{s}(\nu)$ for each $s \in(q, p)$. Then $|\phi(t)|^{s} \log |\phi(t)|$ and $\psi(t) J^{s} \phi(t)$ are jointly continuous $L^{1}(v)-$ valued functions of $t$ and $s$.

To prove Lemma 1, we will use a slightly strengthened version of the dominated convergence theorem. The theorem we use follows from Theorem 16, Chapter 4, of Royden [6] by a subsequence argument.

Modified Dominated Convergence Theorem. Let $\left\{f_{m}\right\}, m=1,2$, $3, \ldots$, and $f$ be measurable functions on $X$ such that $f_{m}(x) \rightarrow f(x)$ a.e. [ $\left.\nu\right]$. Suppose there exist non-negative measurable functions $\left\{g_{m}\right\}, m=1,2$, $3, \ldots$, and $g$ on $X$ with $\left|f_{m}(x)\right| \leqslant g_{m}(x)$ a.e. [ $\left.\nu\right]$ and $g_{m} \rightarrow g$ in $L^{1}(\nu)$. Then $f_{m} \rightarrow f$ in $L^{1}(v)$.

Proof of Lemama 1. Set $G(t, s)=|\phi(t)|^{s} \log |\phi(t)|$. Let $t_{m} \rightarrow t$ in $(a, b)$ and $s_{m} \rightarrow s$ in $(q, p)$. We will show $G\left(t_{m}, s_{m}\right) \rightarrow G(t, s)$ in $L^{1}(v)$. Suppose not. By passing to a subsequence, we may assume $\phi\left(t_{m}\right)(x) \rightarrow \phi(t)(x)$ for almost all $x \in X$; and so $G\left(t_{m}, s_{m}\right)(x) \rightarrow G(t, s)(x)$ for almost all $x \in X$.

Let $\beta, \alpha>0$ be such that $q<s-\beta-\alpha$ and $s+\beta+\alpha<p$. For $m$ sufficiently large that $\left|s_{m}-s\right| \leqslant \beta$, it follows from the estimate (9) for $\log |\phi|$ that

$$
\left|G\left(t_{m}, s_{m}\right)\right|<(e \alpha)^{-1}\left[\left|\phi\left(t_{m}\right)\right|^{s-\beta-\alpha}+\left|\phi\left(t_{m}\right)\right|^{s+\beta+\alpha}\right] .
$$


Now, by hypothesis $\phi\left(t_{m}\right) \rightarrow \phi(t)$ in both $L^{s-\beta-\alpha}$ and $L^{s+\beta+\alpha}$; and so the functions $\left|\phi\left(t_{m}\right)\right|^{s-\beta-\alpha}+\left|\phi\left(t_{m}\right)\right|^{s+\beta+\alpha}$ converge to $|\phi(t)|^{s-\beta-\alpha}+|\phi(t)|^{s+\beta+\alpha}$ in $L^{1}(v)$. The modified Dominated Convergence Theorem implies that $G\left(t_{m}, s_{m}\right) \rightarrow G(t, s)$ in $L^{1}(\nu)$.

Thus the assumption that $G\left(t_{m}, s_{m}\right)$ does not converge to $G(t, s)$ leads to a contradiction. Continuity of $\psi(t) J^{s} \phi(t)$ is proved similarly. This completes the proof of Lemma 1, and therefore of Proposition 1.

5. Gross' inequality. Gross [3] simplified Nelson's proof of the hypercontractive estimates for the Hermite semigroup $e^{-t H}$ by showing them to be equivalent to the logarithmic Sobolev inequality

$$
\int|\phi|^{q} \log |\phi| d \mu \leqslant \frac{q}{2(q-1)} \operatorname{Re}\left\langle H \phi, J^{q} \phi\right\rangle+\|\phi\|_{q}^{q} \log \|\phi\|_{q},
$$

where $\phi$ is in $D_{q}(H), 1<q<\infty$. Here, the norms and the sesquilinear form are taken with respect to the Gauss measure $d \mu(x)=\pi^{-1 / 2} \exp \left(-x^{2}\right) d x$ on $R$. Moreover, he showed that it suffices to show that (10) is true at $q=2$ in order to deduce it in general.

Also, Gross gave an independent proof of (10), thereby providing a new proof of Nelson's estimates.

Observe that for $\phi$ not identically 0 , Gross' inequality can be written

$$
\frac{d}{d q} \log \|\phi\|_{q}^{q} \leqslant \frac{q}{2(q-1)} \cdot \frac{\operatorname{Re}\left\langle H \phi, J^{q} \phi\right\rangle}{\|\phi\|_{q}^{q}}+\log \|\phi\|_{q} .
$$

In this form the similarity with (4) of Theorem 2 above is rather striking. Both (4) and (11) give an estimate for the derivative of the convex function $q \mapsto \log \|\phi\|_{q}^{q}$ (though in (4) the norm is taken with respect to Lebesgue measure). Consequently, I will refer to (4) as a differential (Sobolev) inequality.

The basic idea behind Gross' proof of the equivalence of Nelson's estimates with (10) is to differentiate the inequality $\left\|e^{-t H} \phi\right\|_{p(t, q)} \leqslant\|\phi\|_{q}$ at $t=0$. (10) is simply a restatement of the fact that

$$
\left.\frac{\partial}{\partial t}\right|_{t=0}\left\|e^{-t H} \phi\right\|_{p(t, q)}=\lim _{t \downarrow 0} t^{-1}\left[\left\|e^{-t H} \phi\right\|_{p(t, q)}-\|\phi\|_{q}\right]<0 .
$$

That (10) implies Nelson's estimates is proved by integrating an inequality derivable from (10). See Gross [3, Theorems 1 and 2].

The proof of Theorem 2 in the next section includes many of Gross' arguments adapted to the context of the heat-diffusion semigroup.

6. Proof of Theorem 2. In this section we adopt the notation of Theorem 2. In particular, $L^{q}$ norms are taken with respect to Lebesgue measure on $R^{n}$. Also, $q^{\prime}$ denotes the exponent conjugate to $q$. We prove Theorem 2 by a sequence of lemmas and propositions. 
As we have suggested, the key idea will be to differentiate the expression $\left\|e^{t \Delta} \phi\right\|_{p(t)}$ (where $p(0)=q$ ) at $t=0$. Proposition 1 allows us to do that (for suitable curves $p(t))$, but only if $e^{t \Delta} \phi$ is differentiable at $t=0$ in all $L^{s}$ spaces for $s$ in a neighborhood of $q$. Thus it is natural first to prove that the differential inequality (4) holds at a given $q$ for those $\phi$ which are in $D_{s}(\Delta)$ for all $s$ in a neighborhood of $q$. For such $\phi, d / d q\left(\log \|\phi\|_{q}^{q}\right)$ has a clear meaning since $\phi$ is in $L^{s}, s$ in a neighborhood of $q$.

In the next section we will show that, in fact, (4) holds at a given $q$ for all $\phi \in D_{q}(\Delta)$; and, in particular, we will interpret the expression $d / d q\left(\log \|\phi\|_{q}^{q}\right)$ for such $\phi$.

Proposition 2. Fix $q$ with $1<q<\infty$. Let $\varepsilon>0$ and suppose $\phi \neq 0$ is in $D_{s}(\Delta)$ for all $s \in(q-\varepsilon, q+\varepsilon)$. Assume the norm inequality (1), i.e. $\left\|e^{t \Delta} \phi\right\|_{p}<$ $\left[C_{q} C_{p^{\prime}}\right]^{n}(4 \pi t r)^{-n / 2 r}\|\phi\|_{q}$, holds with this $\phi$ and $q$ for $p \in(q, q+\varepsilon)$ and $t>0$. Then the differential inequality (4) holds for $\phi$ and $q$.

Proof. Let $p(t)$ be a continuously differentiable function $[0, a) \rightarrow[q, q+$ $\varepsilon$ ) with $p(0)=q$ and $p(t)>q$ for $t>0$. Also, we require that the derivative of $p(t)$ at $t=0$, which we denote by $\gamma$, be positive. For $t \in[0, a)$ define $m(t)$ by

$$
m(t)= \begin{cases}{\left[C_{q} C_{p^{\prime}}\right]^{n}(4 \pi t r)^{-n / 2 r},} & t \in(0, a), \\ 1, & t=0,\end{cases}
$$

where $p$ stands for $p(t)$ and $r^{-1}=q^{-1}-p^{-1}$. Straightforward applications of l'Hôpital's rule and the mean value theorem show that $m(t)$ is continuously differentiable on $[0, a)$ with

$$
\left.\frac{d}{d t}\right|_{t=0} \log m(t)=\frac{n \gamma}{2 q^{2}} \log \left[\frac{\gamma}{4 \pi e^{2}(q-1)}\right] .
$$

Furthermore, by Proposition 1 on differentiability (and the chain rule) it follows that $\left\|e^{t \Delta} \phi\right\|_{p(t)}$ is continuously differentiable on $[0, a)$ with

$$
\begin{aligned}
\frac{d}{d t} \log \left\|e^{t \Delta} \phi\right\|_{p(t)}= & \frac{\operatorname{Re}\left\langle\Delta e^{t \Delta} \phi, J^{p} e^{t \Delta} \phi\right\rangle}{\left\|e^{t \Delta} \phi\right\|_{p}^{p}} \\
& +\frac{1}{p} \frac{d p}{d t}\left[\left(\left.\frac{\partial}{\partial s}\right|_{s=p} \log \left\|e^{t \Delta} \phi\right\|_{s}^{s}\right)-\log \left\|e^{t \Delta} \phi\right\|_{p}\right]
\end{aligned}
$$

where $p$ stands for $p(t)$. In particular, at $t=0$ we get 


$$
\begin{aligned}
\left.\frac{d}{d t}\right|_{t=0} \log \left\|e^{t \Delta} \phi\right\|_{p(t)}= & \frac{\operatorname{Re}\left\langle\Delta \phi, J^{q} \phi\right\rangle}{\|\phi\|_{q}^{q}} \\
& +\frac{\gamma}{q}\left[\left(\left.\frac{d}{d s}\right|_{s=q} \log \|\phi\|_{s}^{s}\right)-\log \|\phi\|_{q}\right] .
\end{aligned}
$$

(In applying Proposition 1 to get differentiability at $t=0$, one must smoothly extend $p(t)$ and $e^{t \Delta} \phi$ to small negative values of $t$.)

The hypotheses of the proposition imply that for $t \in(0, a)$,

$$
t^{-1}\left[\log \left\|e^{t \Delta} \phi\right\|_{p(t)}-\log \|\phi\|_{q}\right] \leqslant t^{-1}[\log m(t)-\log m(0)] ;
$$

and thus, letting $t$ decrease to 0 ,

$$
\left.\frac{d}{d t}\right|_{t=0} \log \left\|e^{t \Delta} \phi\right\|_{p(t)}<\left.\frac{d}{d t}\right|_{t=0} \log m(t) .
$$

Substituting in (12) and (14), and rearranging slightly, we get

$$
\begin{aligned}
d /\left.d s\right|_{s=q} \log \|\phi\|_{s}^{s} \leqslant q / \gamma \cdot \operatorname{Re}\left\langle-\Delta \phi, J^{q} \phi\right\rangle /\|\phi\|_{q}^{q} \\
\\
+(n / 2 q) \log \left[\gamma / 4 \pi e^{2}(q-1)\right]+\log \|\phi\|_{q} .
\end{aligned}
$$

(15) is true for any $\gamma>0$ (appropriate $p(t)$ can always be chosen), and it implies, in particular, that $\operatorname{Re}\left\langle-\Delta \phi, J^{q} \phi\right\rangle$ is strictly positive. If $\operatorname{Re}\left\langle-\Delta \phi, J^{q} \phi\right\rangle$ were not positive, appropriate choice of $\gamma>0$ could make the right-hand side of (15) an arbitrarily large negative number. (That $\operatorname{Re}\left\langle-\Delta \phi, J^{q} \phi\right\rangle \geqslant 0$ also follows from the fact that $e^{t \Delta}$ is a contraction semigroup on $L^{q}$.)

We complete the proof of the proposition by minimizing the right-hand side of (15) with respect to $\gamma$. Indeed, letting

$$
\gamma=2 q^{2} / n \cdot \operatorname{Re}\left\langle-\Delta \phi, J^{q} \phi\right\rangle /\|\phi\|_{q}^{q},
$$

we get the differential inequality (4).

Proposition 3. Fix $q$ with $1<q<\infty$. Let $\varepsilon>0$ and suppose $\phi \neq 0$ is in $D_{s}(\Delta)$ for all $s \in(q-\varepsilon, \infty)$. Assume that the differential inequality (4) holds for any $\phi_{1}=e^{t \Delta} \phi, t>0$, at any value $q_{1}$ such that $q<q_{1}<\infty$. Then the norm inequality (1) holds with the given $\phi$ and $q$ for any $p \in(q, \infty)$ and $t>0$.

Proof. Let $\phi$ and $q$ be as in the statement of the proposition. Choose $p$ with $q<p<\infty$ and let $T>0$. We will show that (1) holds with this $\phi, q, p$, and $T$.

Let $\sigma(t)$ be continuously differentiable on some open interval containing $[0, T]$ with $\sigma(0)=q, \sigma(T)=p$, and $d \sigma / d t$ always positive. (An explicit choice of $\sigma(t)$ will eventually be made.) By Proposition 1 (and the chain rule) it follows that $\left\|e^{t \Delta} \phi\right\|_{\sigma(t)}$ is continuously differentiable on $[0, T]$, and its derivative is given by (13) (with $\sigma(t)$ replacing $p(t)$ ). 
By assumption, (4) is satisfied if $q$ is replaced by $q_{1}=\sigma(t)$ and $\phi$ is replaced by $\phi_{1}=e^{t \Delta} \phi, t \in[0, T]$. Since the right-hand side of (4) is obtained by minimizing the right-hand side of (15), it follows that (15) holds with this $q_{1}$ and $\phi_{1}$ replacing $q$ and $\phi$, for any $\gamma>0$.

In particular, if we use at each $t \in[0, T]$ the choices $q_{1}=\sigma(t), \phi_{1}=e^{t \Delta} \phi$, and $\gamma=d \sigma / d t,(15)$ gives an upper bound for $\partial /\left.\partial s\left(\log \left\|e^{t \Delta} \phi\right\|_{s}^{s}\right)\right|_{s=o(t)}$. If we then replace $\partial /\left.\partial s\left(\log \left\|e^{t \Delta} \phi\right\|_{s}^{s}\right)\right|_{s=\sigma(t)}$ in (13) by this bound, we get that, for $t \in[0, T]$,

$$
\frac{d}{d t} \log \left\|e^{t \Delta} \phi\right\|_{\sigma(t)} \leqslant \frac{n}{2 \sigma^{2}} \frac{d \sigma}{d t} \log \left[\frac{d \sigma}{d t} \cdot \frac{1}{4 \pi e^{2}(\sigma-1)}\right],
$$

where $\sigma$ stands for $\sigma(t)$.

At this point we define $\sigma(t)$ by

$$
\frac{1}{q}-\frac{1}{\sigma(t)}=\left(\frac{1}{q}-\frac{1}{p}\right) \frac{t}{T}=\frac{t}{r T}
$$

(recall $r^{-1}=q^{-1}-p^{-1}$ ), and (16) becomes

$$
\frac{d}{d t} \log \left\|e^{i \Delta} \phi\right\|_{\sigma(t)} \leqslant \frac{n}{2 r T} \log \left[\frac{\sigma^{2}}{4 \pi T r e^{2}(\sigma-1)}\right] .
$$

Finally, we integrate $(17)$ over $[0, T]$ and exponentiate, obtaining the inequality

$$
\left\|e^{T \Delta} \phi\right\|_{p} \leqslant\|\phi\|_{q}(4 \pi T r)^{-n / 2 r} \exp \int_{0}^{T} \frac{n}{2 r T} \log \left[\frac{\sigma^{2}}{e^{2}(\sigma-1)}\right] d t .
$$

This integral is easily evaluated by substituting $\tau=\sigma(t)^{-1}$ as the variable of integration; and performing that integration completes the proof of the proposition.

Proposition 2 shows that the norm estimates (1) with $1<q<p<\infty$ and $t>0$ imply that the differential inequality (4) holds at all $q \in(1, \infty)$, where at each $q$ any function $\phi \neq 0$ which is in $D_{s}(\Delta)$ for all $s$ in a neighborhood of $q$ may be used. Proposition 3 implies the converse. Furthermore, by Proposition 2 the norm estimates (1) in the restricted case where $q=2$ and $\phi>0$ is in $D$ imply that the differential inequality (4) holds at $q=2$ for $\phi>0$ in $\mathscr{D}$. The next step in the proof of Theorem 2 is to show that if the differential inequality (4) holds at $q=2$ with all $\phi>0$ in $\mathscr{D}$, then it holds at each $q \in(1, \infty)$ with any $\phi \neq 0$ which is in $D_{s}(\Delta)$ for all $s$ in a neighborhood of $q$. This will be the content of Proposition 4. First we need two lemmas.

LEMMA 2. Suppose the differential inequality (4) holds with $q=2$ for all $\phi>0$ in $D$ not identically 0 . Then (4) holds with $2\langle q\langle\infty$ for all $\phi>0$ in (1) not identically 0 . 
Proof. For $q=2$ and $\phi \in \mathscr{D}$, (4) can be written

$$
\left.\frac{d}{d s}\right|_{s=2} \log \|\phi\|_{s}^{s}<\frac{n}{4} \log \left[\frac{2}{\pi n e} \cdot \frac{\langle\nabla \phi, \nabla \phi\rangle}{\|\phi\|_{2}^{2}}\right]+\log \|\phi\|_{2} .
$$

We claim that (18) must hold for any nonnegative continuously differentiable function $\psi$ with compact support. To see this, let $\sigma \in \mathcal{D}$ be nonnegative and identically 1 on a neighborhood of the support of $\psi$. Certainly $\sigma e^{t} \psi$ is in $\mathscr{D}$ and is nonnegative; and so by hypothesis (18) is true with $\sigma e^{t} \psi$ in place of $\phi$. That (18) holds with $\psi$ now follows by letting $t$ decrease to 0 . (To see that the left-hand side of (18) converges as $t$ decreases to 0 , write

$$
\left.\frac{d}{d s}\right|_{s=2} \log \left\|\sigma e^{t} \psi\right\|_{s}^{s}=\left\|\sigma e^{t} \psi\right\|_{2}^{-2} \int\left|\sigma e^{t} \psi\right|^{2} \log \left|\sigma e^{t} \psi\right|
$$

and use Lemma 1.) This proves the claim.

Now if $\phi>0$ is in $\mathscr{D}$ and $q>2$, then $\phi^{q / 2}$ is nonnegative, continuously differentiable, and has compact support. Thus we may substitute $\phi^{q / 2}$ into (18). Doing this, and manipulating using the following facts (which are easy to verify):

(a) $\log \left\|\phi^{q / 2}\right\|_{2}=(q / 2) \log \|\phi\|_{q}$;

(b) $\left\|\phi^{q / 2}\right\|_{2}^{2}=\|\phi\|_{q}^{q}$;

$$
\left\langle\nabla\left(\phi^{q / 2}\right), \nabla\left(\phi^{q / 2}\right)\right\rangle=q^{2} / 4(q-1) \cdot\left\langle-\Delta \phi, \phi^{q-1}\right\rangle
$$

$$
=q^{2} / 4(q-1) \cdot \operatorname{Re}\left\langle-\Delta, J^{q} \phi\right\rangle ;
$$$$
\left.\frac{d}{d s}\right|_{s=2} \log \left\|\phi^{q / 2}\right\|_{s}^{s}=\left.\frac{q}{2} \frac{d}{d s}\right|_{s=q} \log \|\phi\|_{s}^{s} ;
$$

we get that (4) holds for the given $\phi$ and $q$.

LEMMa 3. Suppose the differential inequality (4) holds at a fixed $q$ for all $\phi>0$ in $\mathscr{D}$ (not identically 0 ). Then it holds at that $q$ with $\phi$ replaced by $\phi_{1}=e^{t \Delta} \phi$ for all $\phi \geqslant 0$ in $\mathscr{D}$ (not identically 0 ) and $t \geqslant 0$.

Proof. The argument is similar to the first part of the proof of the previous lemma. Let $\sigma_{m}, m=1,2,3, \ldots$, be nonnegative functions in $\mathcal{Q}$ such that

(a) $\sigma_{m}(x)=1$ whenever $|x| \leqslant m$,

(b) $\sigma_{m}(x)=0$ whenever $|x| \geqslant m+1$,

(c) $\left|\sigma_{m}(x)\right|,\left|\nabla \sigma_{m}(x)\right|$, and $\left|\Delta \sigma_{m}(x)\right|$ are all uniformly bounded in $m$ and $x$.

Now we let $\psi_{m}=\sigma_{m} e^{t \Delta} \phi=\sigma_{m} \phi_{1}$. We put $\psi_{m}$ into (4) and verify that everything converges as expected as $m \rightarrow \infty$. (We again must use Lemma 1; and we must also use the fact that the duality map $J^{q}$ is continuous as a map from $L^{q}$ into $L^{q^{\prime}}$.)

Proposition 4. Suppose the differential inequality (4) holds at $q=2$ with all 
$\phi \geqslant 0$ in $\mathscr{D}$ (not identically 0$)$. Then it holds for each $q \in(1, \infty)$ with all $\phi$ (not identically 0$)$ such that for some $\varepsilon>0, \phi \in D_{s}(\Delta), s \in(q-\varepsilon, q+\varepsilon)$.

Proof. Suppose (4) holds at $q=2$ with all $\phi \geqslant 0$ in $\mathscr{D}$ (not identically 0 ). It follows from Lemmas 2 and 3 that (4) holds with any $q \in[2, \infty)$ for all $\phi_{1}=e^{t \Delta} \phi$, where $\phi \geqslant 0$ (not identically 0 ) is in $\mathscr{D}$.

Proposition 3 now implies that the norm inequality (1) holds for all $\phi>0$ in $\mathscr{D}, t>0$, and $2 \leqslant q<p<\infty$. By standard limit arguments, it follows that for $t>0$ and $2 \leqslant q<p<\infty$, (1) holds with all $\phi>0$ in $L^{q}\left(R^{n}\right)$. Now $e^{t \Delta}$ is positively preserving, i.e. $\left|e^{t \Delta} \phi\right| \leqslant e^{t \Delta}|\phi|$, and so the norm estimates (1) hold for all $\phi \in L^{q}$ (with $2 \leqslant q<p<\infty$ and $t>0$ ).

At this point we use the fact that the transpose of the map $e^{t \Delta}: L^{q} \rightarrow L^{p}$ is precisely $e^{t \Delta}: L^{p^{\prime}} \rightarrow L^{q^{\prime}}(1<q<p<\infty)$. Since a transpose map has the same norm as the original map, it follows that the norm estimates (1) hold for all $\phi \in L^{q}$ whenever $1<q<p \leqslant 2$ and $t>0$.

Thus we have shown that (1) holds for all $\phi \in L^{q}$ and $t>0$ if either $1<q<p \leqslant 2$ or $2 \leqslant q<p<\infty$. By Proposition 2, this suffices to show that (4) holds for all $q \in(1, \infty)$ with all appropriate $\phi$.

Proposition 4 is now proved; and so is Theorem 2, except for showing that (4) holds whenever $\phi \in D_{q}(\Delta)$.

7. Proof of Theorem 2 (conclusion). Fix $q \in(1, \infty)$ and $\phi \in D_{q}(\Delta)$, not identically 0 . We will show that (4) holds at this $q$ with this $\phi$.

Since $\phi \in D_{q}(\Delta)$, it follows that for some $\varepsilon>0, \phi \in L^{s}\left(R^{n}\right)$ for all $s \in[q, q+\varepsilon)$. To see this, note that for some $\psi \in L^{q}$,

$$
\phi=(1-\Delta)^{-1} \psi=\int_{0}^{\infty} e^{-t} e^{t} \psi d t,
$$

and use (1) to estimate the $L^{s}$ norm of the integral. (This argument lets us avoid appealing directly to the Sobolev inequalities.) Thus, if we let $f(s)=$ $\log \|\phi\|_{s}^{s}$, then $f$ is continuous on $[q, q+\varepsilon)$ and convex on $(q, q+\varepsilon)$. Consequently, the derivative $f^{\prime}(q)$ exists at least as a right-handed derivative, if we allow $f^{\prime}(q)=-\infty$ as a possibility; and $f^{\prime}(q)$ is the limit as $s$ decreases to $q$ of $f^{\prime}(s)$.

Now for $t>0, e^{t \Delta} \phi$ is in $D_{s}(\Delta)$ for all $s>q$. Thus (4) holds at each $s>q$ with all the functions $e^{t \Delta} \phi, t>0$, i.e.,

$$
\begin{aligned}
\frac{\partial}{\partial s} \log \left\|e^{t \Delta} \phi\right\|_{s}^{s} \leqslant & \frac{n}{2 s} \log \left[\frac{s^{2}}{2 \pi n e(s-1)} \cdot \frac{\operatorname{Re}\left\langle-\Delta e^{t \Delta} \phi, J^{s} e^{t \Delta} \phi\right\rangle}{\left\|e^{t \Delta} \phi\right\|_{s}^{s}}\right] \\
& +\log \left\|e^{t \Delta} \phi\right\|_{s}
\end{aligned}
$$

Let $t \downarrow 0$. Then for $s \in(q, q+\varepsilon), e^{t \Delta} \phi$ converges to $\phi$ in $L^{s}$. Also, writing the left-hand side of (19) as $\left\|e^{i \Delta} \phi\right\|_{s}^{-s} \int\left|e^{t \Delta} \phi\right|^{s} \log \left|e^{t \Delta} \phi\right|$, we see from Lemma 1 that 
it converges to $f^{\prime}(s)=\|\phi\|_{s}^{-s} \int|\phi|^{s} \log |\phi|$. Moreover, for $s$ sufficiently close to $q$, $e^{i \Delta} \phi$ converges to $\phi$ in $L^{(s-1) q^{\prime}}$; and since $J^{s}: L^{(s-1) q^{\prime}} \rightarrow L^{q^{\prime}}$ is continuous, $J^{s} e^{i \Delta} \phi$ converges to $J^{s} \phi$ in $L^{q}$. Finally, since $\Delta e^{t \Delta} \phi$ converges to $\Delta \phi$ in $L^{q}$, we get that $\left\langle-\Delta e^{i \Delta} \phi, J^{s} e^{i \Delta} \phi\right\rangle$ converges to $\left\langle-\Delta \phi, J^{s} \phi\right\rangle$ as $t \downarrow 0$.

We have shown that letting $t$ decrease to 0 in (19) leads to the inequality

$$
f^{\prime}(s) \leqslant \frac{n}{2 s} \log \left[\frac{s^{2}}{2 \pi n e(s-1)} \cdot \frac{\operatorname{Re}\left\langle-\Delta \phi, J^{s} \phi\right\rangle}{\|\phi\|_{s}^{s}}\right]+\log \|\phi\|_{s} .
$$

Letting $s$ decrease to $q$, we get the desired result. This completely proves Theorem 2.

8. Comparison with Sobolev-Nirenberg inequalities. In this section we show that the Sobolev-Nirenberg inequalities are in some sense stronger than the differential inequality (4). We do this by transforming one of the Sobolev inequalities so that it is more easily compared with (4).

Proposition 5. The following statements are equivalent:

(i) The Sobolev inequality on $R^{n}$,

$$
\|\phi\|_{r} \leqslant C\|\nabla \phi\|_{2}^{a}\|\phi\|_{2}^{1-a},
$$

holds for all $\phi \geqslant 0$ in $\mathscr{D}$, where $a=n(1 / 2-1 / r)$, whenever $0<a<1$ and $2<r<\infty$.

(ii) The inequality

$$
\begin{aligned}
& \frac{\log \|\phi\|_{p}^{p}-\log \|\phi\|_{q}^{q}}{p-q} \\
& \quad<\frac{n}{2 q} \log \left[\frac{C^{2 / a} q^{2}}{4(q-1)} \cdot \frac{\left\langle-\Delta \phi, J^{q} \phi\right\rangle}{\|\phi\|_{q}^{q}}\right]+\log \|\phi\|_{q}
\end{aligned}
$$

holds for all $\phi \geqslant 0$ in $\mathscr{D}$, where $a=n(1 / 2-q / 2 p)$, whenever $0<a \leqslant 1$ and $2<q<p<\infty$.

The constant $C$ is the same in both (21) and (22) and depends only on the value of $a$. (Norms are with respect to Lebesgue measure on $R^{n}$.)

Proof. Suppose the Sobolev inequality (21) holds for all $\phi>0$ in $\mathscr{D}$. Then it also holds for all $\psi \geqslant 0$ which are continuously differentiable and have compact support. In particular, it holds for $\psi=\phi^{q / 2}$ for all $q \in[2, \infty)$ and $\phi \geqslant 0$ in $\mathscr{D}$.

Making this substitution, and using the fact that

$$
\left\|\nabla\left(\phi^{q / 2}\right)\right\|_{2}^{2}=\left\langle\nabla\left(\phi^{q / 2}\right), \nabla\left(\phi^{q / 2}\right)\right\rangle=q^{2}[4(q-1)]^{-1}\left\langle-\Delta \phi, J^{q} \phi\right\rangle,
$$

we see that for $2 \leqslant q<\infty$ the inequality 


$$
\|\phi\|_{r q / 2}^{q / 2}<C\left[q^{2} / 4(q-1) \cdot\left\langle-\Delta \phi, J^{q} \phi\right\rangle\right]^{a / 2}\|\phi\|_{q}^{(1-a) q / 2}
$$

holds for all $\phi \geqslant 0$ in $\mathscr{D}$, with $a$ and $r$ as in (i) of the proposition.

Let $p=r q / 2$. Then $a=n(1 / 2-q / 2 p)$ and $q<p<\infty$. We successively perform the following manipulations of (23):

(a) raise everything to the $r$ th power,

(b) divide by $\|\phi\|_{q}^{q}$,

(c) take the logarithm,

(d) divide by $p-q$.

The resulting inequality is precisely (22). Thus (i) implies (ii).

The reverse implication follows by letting $q=2$ in (22) and reversing the above manipulations. This proves the proposition.

Observe that (22) provides a nice interpretation of the Sobolev inequality (21) as a bound for the slope of a chord on the graph of the convex function $f(q)=\log \|\phi\|_{q}^{q}$. The differential inequality (4), however, is only an estimate for the derivative of $f$ at the left endpoint of that chord. Thus, since $f$ is convex, (21) is in some sense stronger than (4).

More precisely, (22) implies

$$
\frac{d}{d q} \log \|\phi\|_{q}^{q} \leqslant \frac{n}{2 q} \log \left[\frac{C_{1} q^{2}}{4(q-1)} \cdot \frac{\left\langle-\Delta \phi, J^{q} \phi\right\rangle}{\|\phi\|_{q}^{q}}\right]+\log \|\phi\|_{q},
$$

where $C_{1}=\inf C^{2 / a}$, the infimum being taken over all $a$ allowed in (22) (recall $C$ depends on $a$ ). (24) certainly has the same form as (4); and by the arguments used in $\$ 6$ one can deduce from (24) norm estimates having the same form as (1). However, whether or not (24) is the same as (4), i.e. whether or not the Sobolev inequality (21) implies the differential inequality (4), depends on the value of $C_{1}$.

9. Remarks. Theorems 1 and 2, along with Gross' results [3, Theorems 1 and 2], show that the following are all equivalent:

(a) Nelson's hypercontractive estimates for the Hermite semigroup,

(b) Gross' logarithmic Sobolev inequality,

(c) norm estimates (1) for the heat-diffusion semigroup,

(d) the differential inequality (4).

As we have already mentioned, the norm inequalities (1) for the heatdiffusion semigroup follow from Beckner's sharp convolution inequality [1, Theorem 3, p. 169]. Before he proves the convolution inequality, Beckner proves a sharp inequality for the norm of the Fourier transform $L^{p} \rightarrow L^{p^{\prime}}$, $1<p<2$. From this, one can quickly deduce the sharp convolution inequality

$$
\|\psi * \phi\|_{p} \leqslant\left[C_{r} C_{q} C_{p^{\prime}}\right]^{n}\|\psi\|_{\|}\|\phi\|_{q}
$$


where $p^{-1}=q^{-1}+r^{-1}-1$ with the additional restriction that $1<q, r<2$ and $2<p<\infty$. (See [1, Theorem $3^{\prime}$, p. 169].)

This restricted convolution inequality is sufficient to prove the norm estimates (1) for the heat-diffusion semigroup in the special case that $q=2$. Thus, by Theorem 2, the sharp Fourier transform inequality implies the four facts listed above.

\section{REFERENCES}

1. W. Beckner, Inequalities in Fourier analysis, Ann. of Math. (2) 102 (1975), 159-182.

2. H. J. Brascamp and E. H. Lieb, Best constants in Young's inequality, its converse, and its generalization to more than three functions, Advances in Math. 20 (1976), 151-173.

3. L. Gross, Logarithmic Sobolev inequalities, Amer. J. Math. 97 (1975), 1061-1083.

4. S. Mazur, Uber schwache Konvergence in den Räumen $\left(L^{p}\right)$, Studia Math. 4 (1933), 128-133.

5. E. Nelson, The free Markoff field, J. Functional Analysis 12 (1973), 211-227.

6. H. L. Royden, Real analysis, Macmillan, New York, 1968.

Department of Mathematics, University of TeXas, Austin, TeXas 78712 